\title{
团簇动态相变催化
}

杨金龙

中国科学技术大学, 合肥微尺度物质科学国家研究中心, 合肥 230026

\section{Phase Transition Catalysis of Dynamic Clusters}

\section{Jinlong Yang}

Hefei National Laboratory of Physical Sciences at the Microscale, University of Science and Technology of China,

Hefei 230026, P. R. China.

Email: jlyang@ustc.edu.cn.

Published online: December 19, 2019.

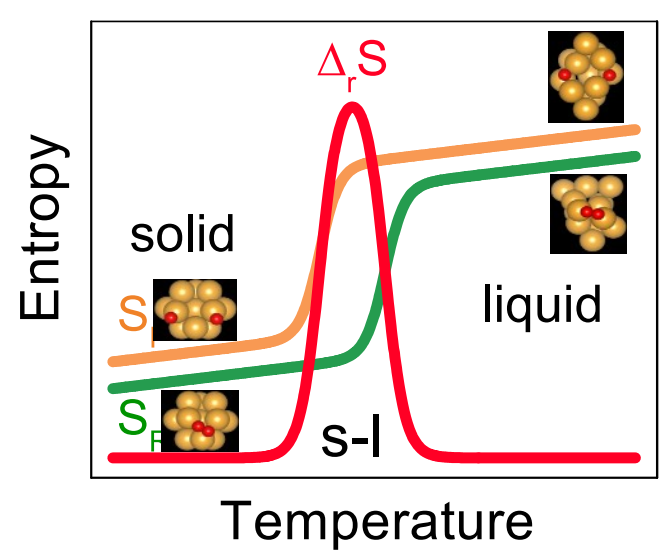

团簇固液相变引起的异常反应摘的变化。

识别和表征催化活性位点对于理解活性的起 源至关重要。随着原位光谱, 显微成像技术以及理 论计算的发展使我们可以得到几个原子特殊排列 组成的活性位的构效关系。然而, 在探索更具挑战 性的催化剂的活性位点方面研究进展甚微, 对于 这些催化剂来说, 催化剂结构对环境极度敏感, 在 反应过程中结构是处于不断变化的 ${ }^{1-4}$ 。当催化剂 动态结构演化的时间尺度(毫秒至秒)远慢于基元 反应发生的时间尺度(皮秒)时, 在基元反应过程中 催化剂活性位结构仍然可被认为是静态的。但当 催化剂结构动态变化的时间尺度与化学反应的时 间尺度一致时, 与反应耦合的催化剂的动态结构 波动对反应会产生怎样的影响呢? 在此之前还未 有研究工作从这一角度来阐述催化剂的动态催化 效应。
厦门大学程俊教授课题组首次以金团簇为 例, 通过第一性原理分子动力学模拟和自由能计 算方法阐明了与反应耦合的催化剂的动态结构波 动对反应的影响。通过准确计算不同温度下的反 应自由能变化和能垒, 发现反应自由能和能垒均 随温度升高异常降低, 并且观测到反应商变在特 定的温度区间呈现为具有巨大峰值的类高斯分 布。进一步分析团簇催化剂的相变行为发现反应 物种的不同吸附状态(反应物, 过渡态以及产物)会 影响团簇的相变温度, 进而导致团簇在特定温度 区间发生准一级固液相变行为, 这一相变过程带 来的巨大熵增使得反应自由能骤降, 推动反应进 行。

同时, 这一现象也存在于实际的负载催化体 系 $\mathrm{Au} / \mathrm{MgO}$, 表明这一理论计算发现的团簇动态相 
变催化很可能广泛存在于实际催化过程中。并且 通过研究不同反应表明相变催化效应与反应类型 相关，因此其不仅影响反应活性也对反应选择性 有影响。

上述研究成果近期在Nature Communications 期刊上在线发表 ${ }^{5}$ 。这一研究工作创新性地提出局 域化学反应与广域环境(含催化材料)的复杂长程 耦合作用的新诠释。目前对化学反应的普遍看法 是它们是 “局部的”, 并且只能受邻近的配位环境 的影响, 因为化学键的性质是短程的。令人惊奇的 是, 他们工作表明通过动态团簇的固-液相转变机 理, 造成构象熵的大幅变化, 可以从全局上影响基 元反应。这一理论发现有可能被相关实验验证, 预 期可以引起实验学家的研究兴趣。这一发现也可 能为生物酶催化中蛋白质动态结构演化的重要作 用提供新的思路, 即长程的蛋白质动力学在酶催
化中起着的作用。

\section{References}

(1) Wang, Y. G.; Mei, D.; Glezakou, V. A.; Li, J.; Rousseau, R. Nat Commun. 2015, 6, 6511. doi: 10.1038/ncomms 7511

(2) Taccardi, N.; Grabau, M.; Debuschewitz, J.; Distaso, M.; Brandl, M.; Hock, R.; Maier, F.; Papp, C.; Erhard, J.; Neiss, C.; et al. Nat. Chem. 2017, 9, 862. doi: 10.1038/nchem.2822

(3) Naoto, K.; Keju, S.; Ryotaro, A.; Masakazu, T.; Takehiro, T.; Hideto, Y.; Seiji, T. Nat. Commun. 2018, 9, 2060. doi: 10.1038/s41467-018-04412-4

(4) Zandkarimi, B.; Alexandrova, A. N. Wiley Interdiscip. Rev. Comput Mol. Sci. 2019, 9, e1420. doi: 10.1002/wcms. 1420

(5) Sun, J.; Cheng, J. Nat. Commun. 2019, 10, 5400. doi: 10.1038/s41467-019-13509-3 